\title{
Miscibility Influence in the Thermal Stability and Kinetic Parameters of Poly (3-hydroxybutyrate)/ Poly (ethylene terephthalate) Sulphonated Blends
}

\author{
Rafael Silva, Gizilene M. Carvalho, Edvani C. Muniz, Adley F. Rubira \\ Departamento de Química, UEM
}

\begin{abstract}
The thermal degradation of miscible and immiscible poly (3-hidroxy butyrate) PHB/ poly (ethylene terephthalate) sulphonated (PETs) blends was investigated using thermogravimetric analyses. Model-free kinetic analysis, Vyazovkin and Flynn-Wall-Ozawa's methods, were used to determine the apparent activation energy in the whole interval of degradation of the pure polymers, immiscible blends, and miscible blends. The thermal stability of both polymers in their blends is higher when compared to the pure polymers. The synergistic effect in the thermal stability in the blends is higher for the miscible blend where the formation of the specific interaction between PHB and PETs occurs. The apparent activation energy of the individual polymers is higher in PETs/PHB blends, and this effect is potentiated by the miscibility of the blend.
\end{abstract}

Keywords: Biodegradable polymer, model-free kinetic analyses, polymeric blends, thermal degradation.

\section{Introduction}

Natural and biodegradable polymers have limited applications due their limited thermal and mechanical properties. In the sense of improving these characteristics, the blend preparation using polymers of high mechanic and thermal performance have been reported in the literature ${ }^{[1-3]}$. The polyhidroxyalkanoates (PHAs) stands out among the natural and biodegradable polymers. PHAs are aliphatic polyesters that can suffer hydrolytic or enzymatic degradation and under the action of microorganisms PHAs degrade completely to carbon dioxide and water ${ }^{[4-7]}$.

PHB, a polyhidroxyalkanoate, presents good barrier properties, biocompatibility, biodegradability, and sensorial proprieties. These features make the application of PHB to food product packaging possible, as proposed by several authors ${ }^{[8-9]}$. In the literature, total or partially miscible blends of PHB were reported ${ }^{[10-12]}$. Among the blends reported in the literature can be emphasized the PETs/PHB blends, reported by our group ${ }^{[13]}$, and that were used in the present work. Miscible blends of PETs/PHB are obtained when specific interactions between PHB and PETs are formed. In the absence of these interactions the blends are immiscible. The formation of the specific interactions between PHB and PETs depends on of the PETs conformation in solution (blends produced by the "casting" method) because PETs in solvents of low polarity tends to form cluster with ionic groups inside, what hinders the carrying out of the specific interactions. The chemical aspects of the miscibility for these blends are discussed in previous work ${ }^{[13]}$.

The knowledge of the thermal stability and of the kinetic parameters of the thermal degradation process of polymers and their blends is important in the processing and application of the same ones ${ }^{[14,15]}$. The determination of the kinetic parameters can be made by different methods, and the main ones use thermogravimetric techniques ${ }^{[16-18]}$.

In the present work is reported the study of the thermal degradation behavior of pure PHB, pure PETs and of mixtures of PHB with PETs in miscible and immiscible blends as well as the effect of blend miscibility on the apparent activation energy of the thermal degradation process. The apparent activation energy is determined by two different model-free kinetic analyses, Vyazovkin and Flynn-Wall-Ozawa's methods. Model-free kinetic method was applied due to the possibility of analysis of the apparent activation energy variation in function of the degradation process extent; this characteristic allowed the individual study of polymers behavior in the blends.

\section{Experimental}

\section{Materials}

The water soluble sulphonated PET ionomer (PETs) under the trade name of Gerol-PS- $20^{\circledR}$ was obtained from Rhodia (20\% sulphonated) and PHB was donated by Biocycle. PHB was purified by successive recrystallization using chloroform (Nuclear) $(3 \% \mathrm{w} / \mathrm{v})$ as a solvent and petroleum ether (Chemco) as a non-solvent. The polymeric materials, pure polymers and blends, were used in the form of films, produced by the "casting" method, using dichloromethane as solvent and dry at room temperature $\left( \pm 25^{\circ} \mathrm{C}\right)$ for 3 hours.

The compositions of the immiscible (blend 1) and miscible (blend 2) blends, in weight fractions, are $0.35 / 0.65$ and $0.81 / 0.19$, respectively, in relationship of PETs for PHB. The obtaining and characterization process of these blends were reported previously by our group ${ }^{[13]}$. 


\section{Thermogravimetric analysis}

The thermogravimetric analyses were carried out in Shimadzu TGA-50 thermogravimetric analyser with heating rates of $2.5,5$ and $15{ }^{\circ} \mathrm{C} / \mathrm{min}$. The measures were accomplished with flow of nitrogen of $20 \mathrm{ml} / \mathrm{min}$. The samples with approximately weight of $6.0 \mathrm{mg}$ were decomposed in a platinum pan.

\section{Kinetic analysis}

The kinetic behavior of the thermal degradation of a polymer can be expressed for Equation 1:

$$
\frac{d \alpha}{d t}=k(T) f(\alpha)
$$

where the reaction rate $(\mathrm{d} \alpha / \mathrm{dt})$ is the product of two functions, one depending only on the absolute temperature, $(\mathrm{T})$ and the other depending only on conversion $(\alpha)$.

The function $k(\mathrm{~T})$ can be substituted by the Equation of Arrhenius (Equation 2):

$$
k(T)=A \exp \left(-\frac{E_{a}}{R T}\right)
$$

where $\mathrm{E}_{\mathrm{a}}$ is the energy of activation of the process, $\mathrm{A}$ is the pre-exponential factor and $\mathrm{R}$ is the universal gas constant.

The Kinetic expression can be transformed into nonisothermal rate expression describing reaction rates as a function of temperature at a constant heating rate $(\beta)$, thus one obtains (Equation 3):

$$
\frac{d \alpha}{d T}=\frac{A}{\beta} \exp \left(-\frac{E_{a}}{R T}\right) f(\alpha)
$$

The function $f(\alpha)$ depends on the mechanism of the decomposition reaction. The mechanisms of polymers decomposition are complex, in some cases more than one decomposition mechanism is possible ${ }^{[19-21]}$. In these cases, the preponderance of a mechanism of decomposition is dependent of the conversion degree and of the temperature.

The activation energy can be determined without the knowledge of $f(\alpha)$ through model-free isoconversional methods. The basic assumption of these methods is that the reaction rate for a constant extent of conversion depends only on the temperature ${ }^{[22-25]}$. An advantage of these methods is that the activation energy can be determined as a function of the conversion. This aspect is important for the analysis of mixtures, for example polymeric blends, which the activation energy changes abruptly in function of the conversion process.

Vyazovkin et al. developed an isoconversional method that allows both simple and complex reactions to be evaluated ${ }^{[26]}$. Integrating up to conversion Equation 3 gives (Equation 4):

$$
\int_{0}^{\alpha} \frac{d \alpha}{f(\alpha)}=\frac{A}{\beta} \int_{T_{0}}^{T} \exp \left(-\frac{E_{a}}{R T}\right) d T=g(\alpha)
$$

since $\mathrm{E}_{\mathrm{a}} / \mathrm{RT} \gg>1$, the temperature integral can be approximated by (Equation 5):

$$
\int_{T_{0}}^{T} \exp \left(-\frac{E_{a}}{R T}\right) d T \approx \frac{R}{E_{a}} T^{2} \exp \left(-\frac{E_{a}}{R T}\right)
$$

substituting the temperature integral and taking the logarithm, one obtains (Equation 6):

$$
\ln \frac{\beta}{T^{2}}=\ln \left[\frac{R A}{E_{a} g(\alpha)}\right]-\frac{E_{a}}{R T_{\alpha}}
$$

to apply this method, it is necessary to obtain at least three different heating rates $(\beta)$, the respective conversion curves being evaluated from the measured TG curves ${ }^{[27]}$. For each conversion $(\alpha)$, the plot of $\left[\ln \left(\beta / \mathrm{T}^{2}\right)\right]$ against [1/RT] should provide a straight line with slope directly proportional to $\mathrm{E}_{\mathrm{a}}$, and thus the activation energy is obtained as a function of conversion.

In order to compare the result of Vyazovkin's method, activation energy may be evaluated by Flynn-Wall-Ozawa method $^{[23,24]}$. As like as Vyazovkin's method the Flynn-Wall-Ozawa's method involve multiple heating rates $(\beta)$ and can be used without the knowledge of the kinetic model, the Flynn-Wall-Ozawa's method gives the Equation 7:

$$
\log \beta=-0.4567 \frac{E_{a}}{R T}-2.315+\log \left(\frac{A E_{a}}{R}\right)-\log g(\alpha)(7)
$$

The dependence of $\log \beta$ on $1 / \mathrm{T}$ for different heating rates at constant degree of conversion $(\alpha)$ represents a straight line with slope directly proportional to $\mathrm{E}_{\mathrm{a}}$.

\section{Result and Discussion}

\section{Analysis thermogravimetric}

The studies of thermo degradation were carried out in inert atmosphere to avoid differences in the diffusibility of the oxidant gas through the polymeric blends with different compositions. The mechanism of the thermo degradation in inert atmosphere of PHB was analyzed by Aoyagi et al. ${ }^{[28]}$, According to Aoyagi et al. the PHB is degraded by a random chain scission.

The curves of thermal decomposition (TG) and their derived curves (DTG) for PHB at different values of $\beta$, are shown in Figure 1. These curves indicate that the reaction of decomposition of PHB happens in a single-stage, in a narrow temperature range. The onset decomposition temperature $\left(\mathrm{T}_{\mathrm{i}}\right)$, as well as the maximum rate decomposition temperature $\left(\mathrm{T}_{\max }\right)$ and the final decomposition temperature $\left(\mathrm{T}_{\mathrm{f}}\right)$ show a shift that depends on $\beta$, as can be seen in Table 1 . This shift is due to the delay in the real sample temperature to the temperature read off by the equipment.

The TG and DTG curves of the thermal composition of PETs at different $\beta$ are shown in Figure 2. The thermal decomposition of PETs happens in two stages, and for $\beta$ equal to $2.5^{\circ} \mathrm{C} / \mathrm{min}$, the first stage begins in $307.2^{\circ} \mathrm{C}$ and it finishes in $395.6^{\circ} \mathrm{C}$, while the second stage begins in $439.9^{\circ} \mathrm{C}$ and 


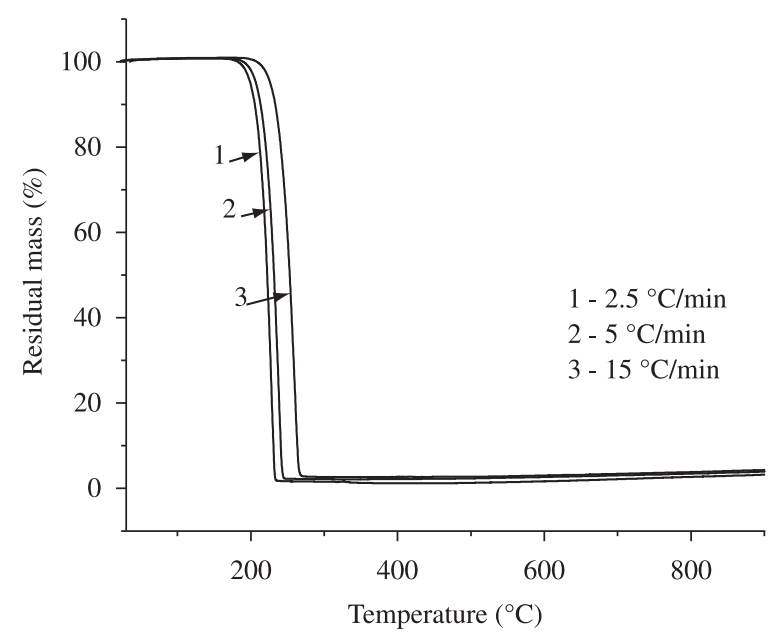

(a)

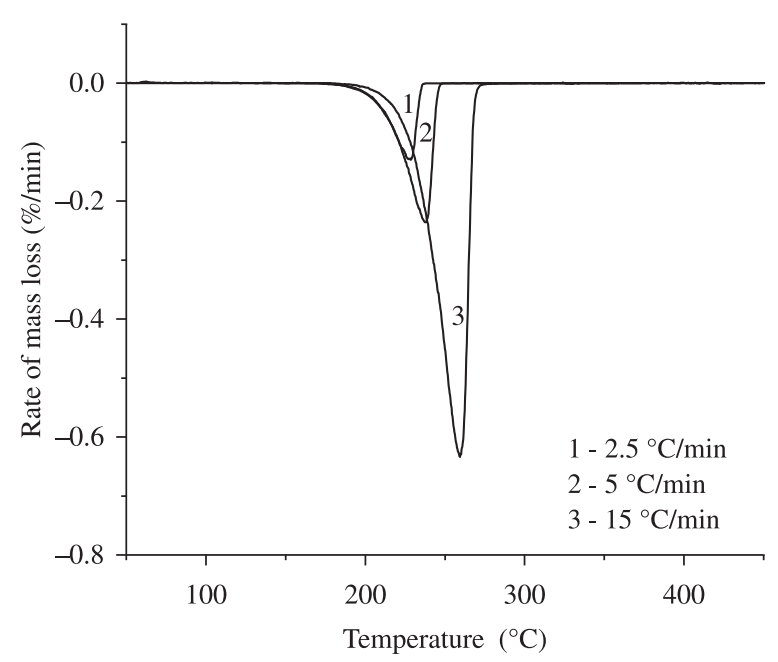

(b)

Figure 1. Curves of TG for PHB in different values of $\beta$ (a) and their derived curves.; DTG (b).

it finishes in $654.3^{\circ} \mathrm{C}$. The values of $\mathrm{T}_{\mathrm{i}}, \mathrm{T}_{\max }$ and $\mathrm{T}_{\mathrm{f}}$ for the two stage of thermal decomposition of PETs at different $\beta$ are shown in Table 1 . The first stage of decomposition of PETs can be attributed to the carbon chain decomposition. The second one to the decomposition of inorganic residues formed due to presence of the group $\mathrm{SO}_{3}^{-}$covalently linked to the polymer. This statement is based in the fact that the PET degrades in only one stage like demonstrated by other authors $^{[29,30]}$.

TG and DTG curves of the blends 1 and 2, in different $\beta$, are shown in the Figures 3 and 4 . It can be verified that the one-stage of PHB degradation and the two-stage PETs degradation happen in different temperature range, it making possible the individual analysis of the polymers in the blends. The second stage of the degradation of PETs cannot be noticed in TG and DTG curves of the blend 1, due to the fact of this blend to be rich in PHB.

Through a more detailed analysis of the values disposed in the Table 1 , it can be affirmed that the $\mathrm{T}_{\mathrm{i}}, \mathrm{T}_{\max }$ and $\mathrm{T}_{\mathrm{f}}$ of PHB and of PETs in both blends are superior to the determined
Table 1. Thermal data obtained from TGA scans of the samples.

\begin{tabular}{|c|c|c|c|c|c|}
\hline Sample & $\beta /{ }^{\circ} \mathbf{C} / \mathbf{m i n}$ & stage & $\mathbf{T}_{\mathbf{i}} /{ }^{\circ} \mathbf{C}$ & $\mathbf{T}_{\max } /{ }^{\circ} \mathbf{C}$ & $\mathrm{T}_{\mathrm{f}} /{ }^{\circ} \mathrm{C}$ \\
\hline \multirow[t]{3}{*}{ PHB } & 2.5 & 1 & 198.0 & 228.4 & 233.0 \\
\hline & 5 & 1 & 207.2 & 236.3 & 243.3 \\
\hline & 15 & 1 & 230.3 & 257.0 & 265.7 \\
\hline \multirow[t]{6}{*}{ PETs } & 2.5 & 1 & 307.2 & 381.7 & 395.6 \\
\hline & & 2 & 439.9 & 529.7 & 654.3 \\
\hline & 5 & 1 & 332.5 & 393.5 & 408.8 \\
\hline & & 2 & 460.7 & 713.0 & 746.2 \\
\hline & 15 & 1 & 343.3 & 402.5 & 431.4 \\
\hline & & 2 & 645.2 & 789.2 & 881.4 \\
\hline \multirow[t]{6}{*}{ Blend 1} & 2.5 & 1 & 244.1 & 262.4 & 269.8 \\
\hline & & 2 & 354.4 & 401.2 & 422.1 \\
\hline & 5 & 1 & 252.4 & 278.2 & 285.2 \\
\hline & & 2 & 372.8 & 420.2 & 433.5 \\
\hline & 15 & 1 & 259.8 & 292.0 & 304.4 \\
\hline & & 2 & 391.8 & 440.0 & 457.9 \\
\hline \multirow[t]{9}{*}{ Blend 2} & 2.5 & 1 & 253.7 & 268.0 & 276.9 \\
\hline & & 2 & 340.5 & 409.0 & 425.5 \\
\hline & & 3 & 636.6 & 671.8 & 712.2 \\
\hline & 5 & 1 & 261.0 & 278.0 & 289.6 \\
\hline & & 2 & 367.1 & 420.6 & 435.9 \\
\hline & & 3 & 641.0 & 701.3 & 831.0 \\
\hline & 15 & 1 & 278.0 & 289.1 & 315.1 \\
\hline & & 2 & 378.8 & 446.1 & 464.8 \\
\hline & & 3 & 699.6 & 748.9 & 952.0 \\
\hline
\end{tabular}

values for pure polymers in all $\beta$ tested. The values of $T_{i}$ and $T_{f}$ for the blend 2 are significantly superiors to the values of the blend 1, mainly in relation to PHB degradation. The values of $\mathrm{T}_{\max }$ are practically equivalent. The addition of PETs to PHB increases the thermal stability of both polymers. However, this effect is more intense for the PHB. The difference in the $T_{i}$ and $T_{f}$ among the blends 1 and 2 can be attributed the miscibility of the mixture, the blend where PHB and PETs possess larger thermal stability is miscible. Therefore, the existence of specific interactions between PHB and PETs increase the effect synergic in the thermal stability. The miscibility or immiscibility of the blends was determined by the variation of the glass transition temperature ${ }^{[13]}$.

From the thermal data can be inferred that the formation of these blends does not cause changes in the polymer degradation mechanisms because the polymers degradations steps can be verified isolated and the effect of the blend formation were observed in both blends, miscible and immiscible. Comparing the degradations steps in the blends, the miscibility causes only differences in the intensity of the temperature shifts. The polymers in the blends, miscible or immiscible, have more alternative ways to dissipate the energy accumulated in the materials with heating process when compared to the pure polymers, such as the increase of the interfacial area and diffusion of chains among the phases. Alternatives pathways for the dissipation of the energy increase the thermal stability of polymers because these ways 


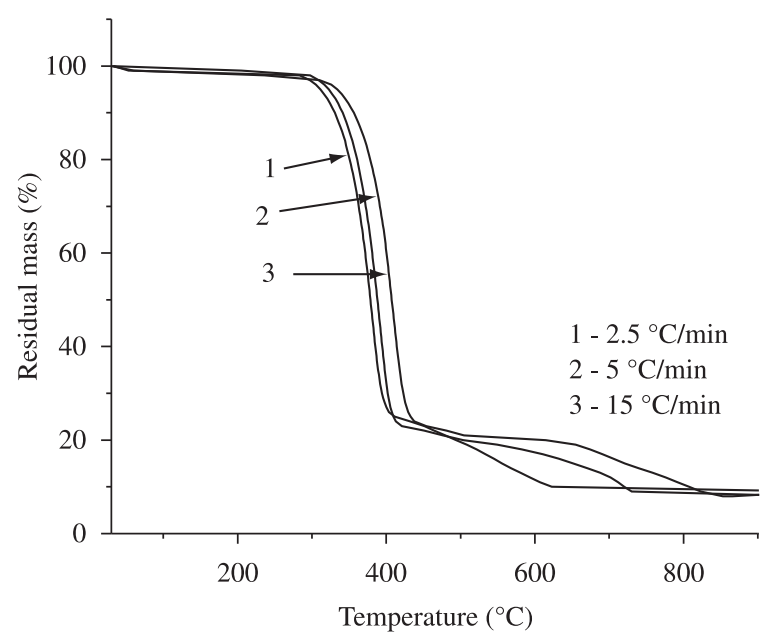

(a)

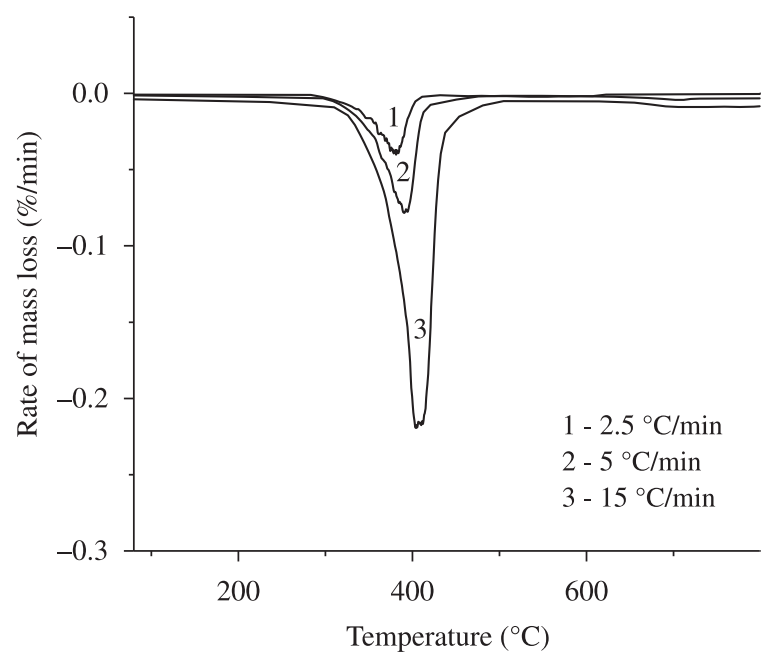

(b)

Figure 2. TG curves for PETs in different values of $\beta$ (a) and their derived curves; DTG (b).

compete to the bonds ruptures by energy transfers during the heating. The miscibility influence in the temperature shift can be associated to the break of the specific interaction existent in the miscible blend. According to the analysis of the blends between PETs and PHB, in miscible blends there is a specific interaction between the carbonyl groups of the PHB and the sulfonate group of the PETs ${ }^{[13]}$.

\section{Kinetic analysis}

Vyazovkin and Flynn-Wall-Ozawa's methods were used to evaluate the variation of apparent activation energy of the decomposition process to the pure polymers and to the polymers in the blends. The apparent activation energy determined by Vyazovkin and Flynn-Wall-Ozawa methods are shown in the Figure 5. The results obtained by both methods presented similar behavior, a slight decrease in the $\mathrm{E}_{\mathrm{a}}$ with the progress of the degradation process. The difference in the numeric results between the methods are smaller than $3 \mathrm{~kJ} \mathrm{~mol}^{-1}$ are, for equivalents process stage. The average activation energy value in the entire process, using

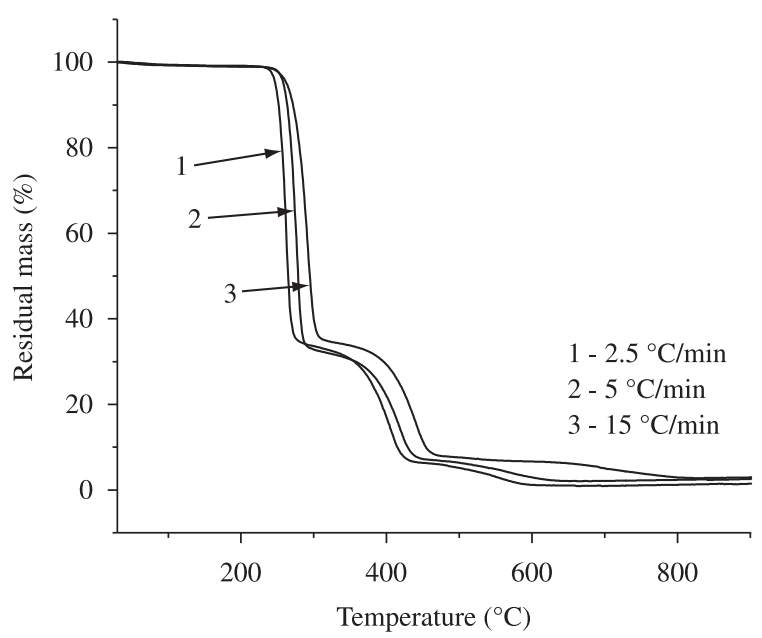

(a)

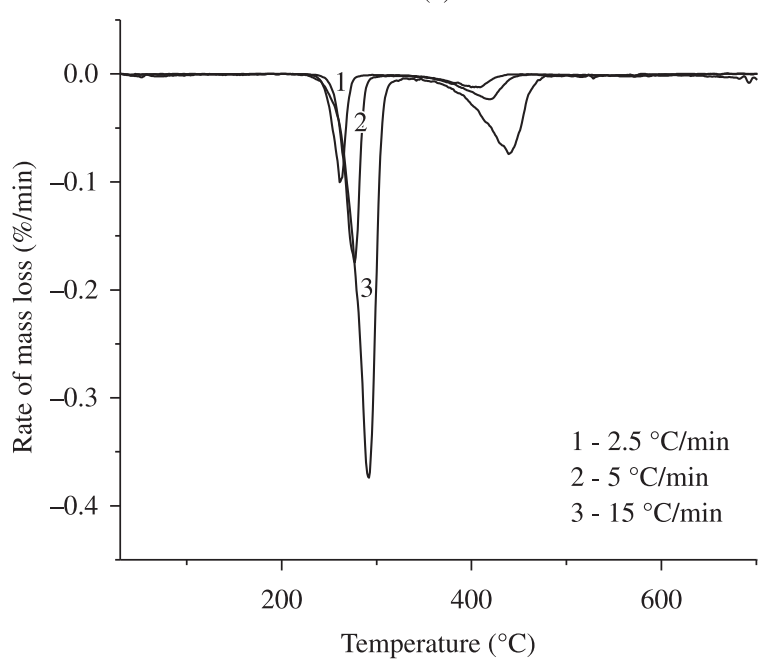

(b)

Figure 3. TG curves for the blend 1 in different values of $\beta$ (a) and their derived curves; DTG (b).

the results of two methods, is $118 \mathrm{~kJ} \cdot \mathrm{mol}^{-1}$. This result is in agreement with the result reported by Matko Erceg et al., ${ }^{[31]}$ which determined through the Kissinger method a medium value of $131 \mathrm{~kJ}^{\mathrm{mol}}{ }^{-1}$ for PHB.

The dependence of the apparent activation energy on conversion for PETs is shown in the Figure 6. The existence of more than one degradation stage for the PETs is easily noted. The kinetic analysis confirms the existence of two stage of thermal degradation. For the first stage the Vyazovkin and Flynn-Wall-Ozawa's methods provide equivalents results. The value of $\mathrm{E}_{\mathrm{a}}$ slightly increases with the increase of $\alpha$ in the 0.10 to 0.80 interval. The average value of $\mathrm{E}_{\mathrm{a}}$ in this conversion interval is $215 \mathrm{~kJ} \mathrm{~mol}^{-1}$. An abrupt decrease in $\mathrm{E}_{\mathrm{a}}$ occurs at nearly $80 \%$ of the conversion, and after this point the $\mathrm{E}_{\mathrm{a}}$ become practically constant indicating the change in the degradation process that are attributed to the degradation of the sulfonate groups linked. The agreement between the both methods used in the second stage as not close as in the first stage. The average values of $\mathrm{E}_{\mathrm{a}}$ were 58 and $70 \mathrm{~kJ}^{\mathrm{mol}}{ }^{-1}$ as 


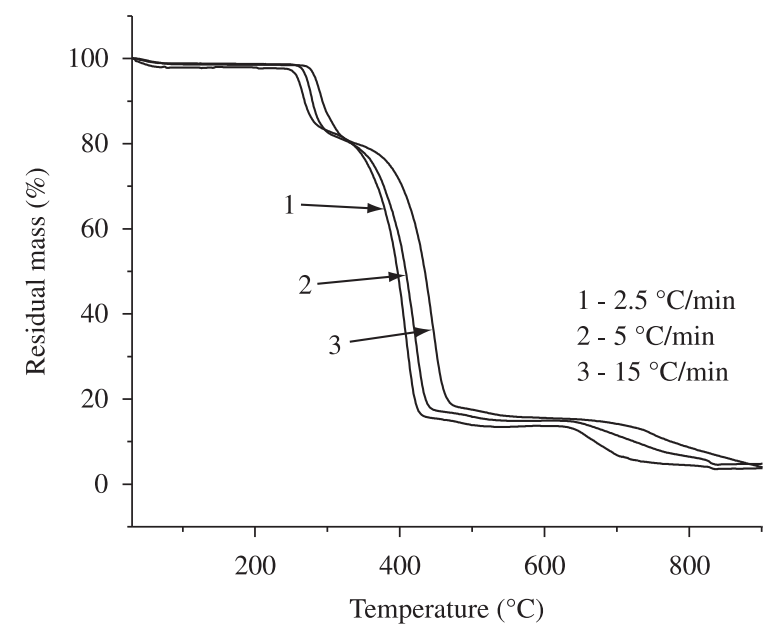

(a)

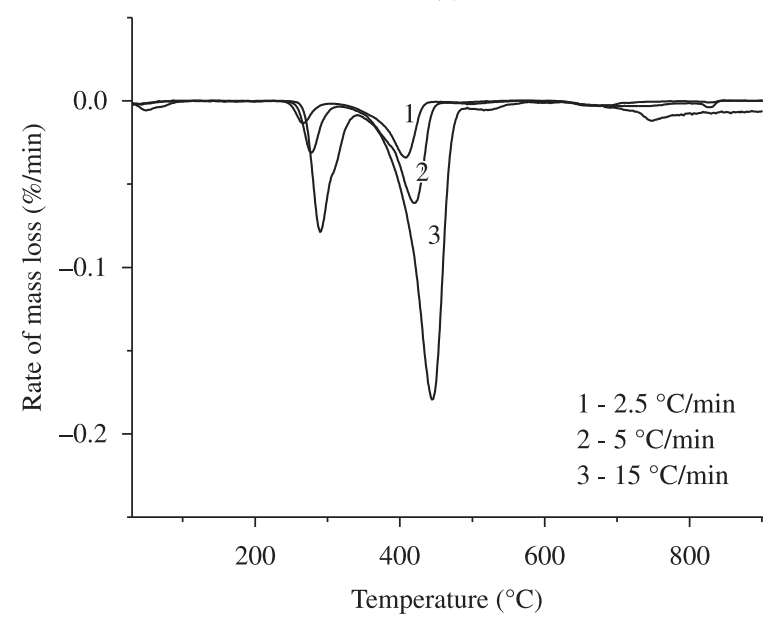

(b)

Figure 4. TG curves for the blend 2 in different values of $\beta$ (a) and their derived curves; DTG (b).

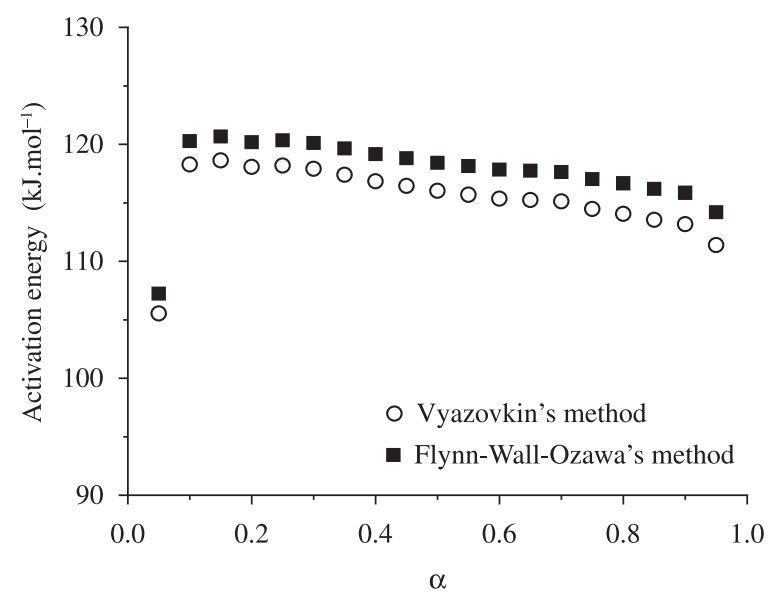

Figure 5. Apparent activation energy in function of conversion for the PHB.

determined by Vyazovkin and Flynn-Wall-Ozawa methods, respectively.

The agreement in the results obtained from both methods for the blends is very close. The apparent activation energy for the blends is not constant in the whole conversion interval. In the Figure 7 can be seen the results of $\mathrm{E}_{\mathrm{a}}$ for the blend 1. The

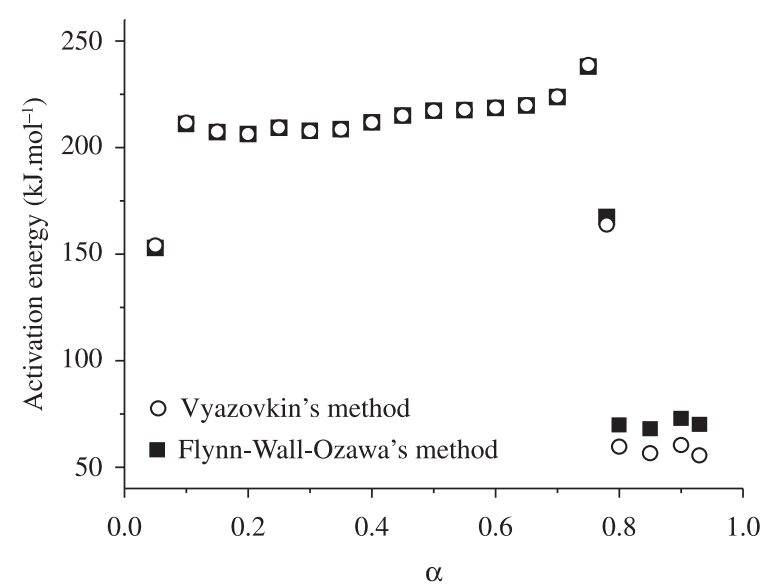

Figure 6. Apparent activation energy in function of conversion for the PETs.

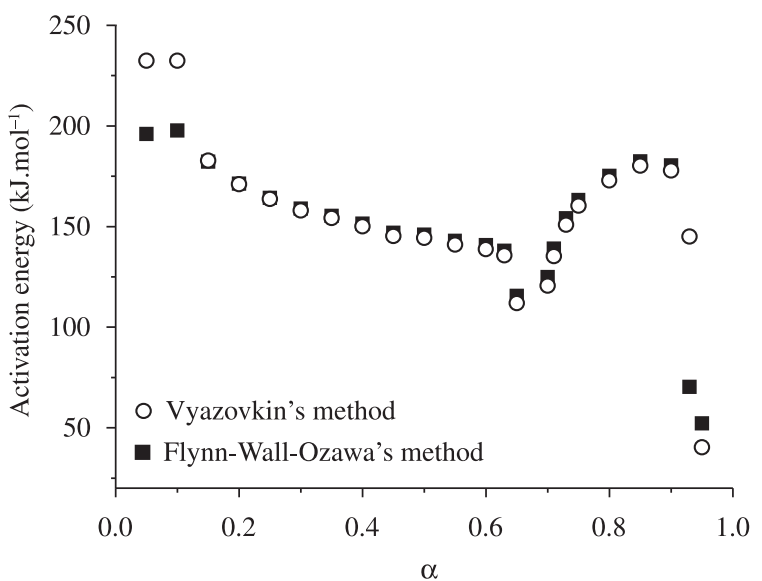

Figure 7. Apparent activation energy in function of conversion for the blend 1.

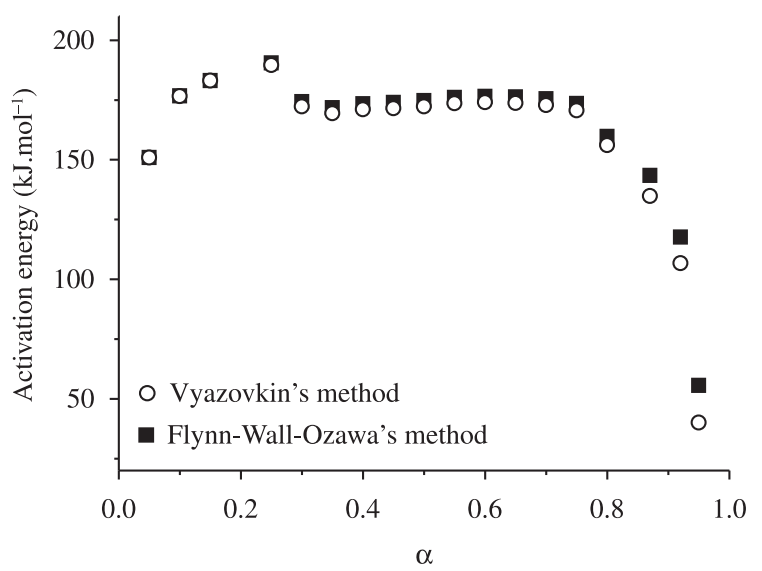

Figure 8. Apparent activation energy in function of conversion for the blend 2.

composition of the blend 1 is $35 \%$ (w/w) of PETs and $65 \%$ $(\mathrm{w} / \mathrm{w})$ of PHB. Thus, the conversion interval ranging from zero until approximately 0.65 is corresponding only to PHB degradation. In this interval the $\mathrm{E}_{\mathrm{a}}$ decrease in function of the conversion process initially higher than 200 to $130 \mathrm{~kJ} . \mathrm{mol}^{-1}$ in $\alpha$ of 0.65 . The apparent activation energy of PHB in the blend 1 is higher than of the pure PHB.

In the blend 1 the conversion $(\alpha)$ referent to PETs occurs after 0.65 . In this interval occurs a quickly increase in the $\mathrm{E}_{\mathrm{a}}$

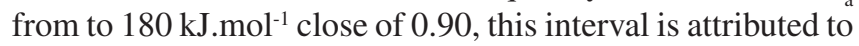


first stage of degradation of PETs. After 0.90 the $\mathrm{E}_{\mathrm{a}}$ decrease abruptly, this should be caused due the existence of second stage of PETs.

The blend 2 consists of $19 \%$ (w/w) of PHB and $81 \%$ (w/w) of PETs. For this blend $\mathrm{E}_{\mathrm{a}}$ increases with the advance of conversion in the interval from zero to 0.20 , as shown in Figure 8. This $\mathrm{E}_{\mathrm{a}}$ values correspond to the degradation of PHB in this blend, and are higher than for pure PHB. The apparent activation energy has linear behavior in the interval of the first stage of PETs decomposition that is going of 0.20 to 0.80 , and $\mathrm{E}_{\mathrm{a}}$ decreases abruptly from 0.80 to 1.00 .

Comparing the two blends, it can be noted that the medium value of $\mathrm{E}_{\mathrm{a}}$ for the PHB is larger for the PHB in the blend 2 than the PHB in the blend 1, the same behavior also occurs for the PETs, which shows a higher average value of $\mathrm{E}_{\mathrm{a}}$ in the blend 2 than in the blend 1 . These facts contributed for the conclusion that the miscibility behavior directly influences the apparent activation energy of the individual polymers in the blend.

\section{Conclusion}

The present study demonstrated that the two different methods, Vyazovkin and Flynn-Wall-Ozawa, provided close values of $\mathrm{E}_{\mathrm{a}}$. The model-free kinetic analysis is an outstanding alternative for estimating the apparent activation energy for the thermal degradation of polymeric blends, and these methods making possible the individual analysis of polymers in the blends.

The formation of blends between PHB and PETs increases the thermal stability of both polymers. The synergism effect in the thermal stability is more accentuated for the miscible blend, probably due to the formation of specific interaction between PHB and PETs. The formation of PETs/PHB blends increases the individual $\mathrm{E}$ of the polymers and this increase is potentiated by the miscibility of the blend.

In addition, the miscibility of polymeric blends influences the apparent activation energy of thermal degradation. This influence may be detected by the kinetic study of TG degradation data.

\section{References}

1. An, Y. X.; Li, L. X.; Dong, L. S.; Mo,.Z. S. \& Feng, L. J. Polym. Sci. Pol. Phys., 37, p.443 (1999).

2. An, Y. X.; Li, L. X.; Dong, L. S.; Mo,.Z. S. \& Feng, L. J. Polym. Sci. Pol. Phys., 38, p.1860 (2000).

3. Yuan, Y. M \& Ruckenstein, E. Polymer, 39, p.1893 (1998).

4. Kellerhals, M. B.; Kessler, B.; Witholt, B.; Tchouboukov, A. \& Brandl, H. - Macromolecules, 33, p.4680 (2000).

5. El-Hadi, A.; Schnabel, R.; Straube, E.; Muller, G. \& Henning S. - Polym. Test., 21, p.665 (2002).

6. Squio, C. R. \& Aragão G. M. F. de. - Quim. Nova, 27, p.615 (2004).
7. Siqueira, A. P. L.; Poley L. H.; Sanchez, R.; Silva, M. G. da \& Vargas H. J. - Phys. IV, 125, p.297 (2005).

8. Bucci, D. Z.; Tavares, L. B. B. \& Sell, I. - Polym. Test., 26, p.235 (2007).

9. Sanchez-Garcia, M. D.; Gimenez, E. \& Lagaron, J. M. J. Appl. Polym. Sci., 108, p.2787 (2008).

10. Blumm, E. \& Owen, A. J. - Polymer, 36, p.4077 (1995).

11. Iriondo, P.; Iurin, J. J. \& Fernandez-Berridi, M. J. Polymer, 36, p.3235 (1995).

12. Zhang, L. L.; Xiong, C. D \& Deng, X. M. - Polymer, 37, p.235 (1996).

13. Silva, R.; Carvalho, G. M.; Muniz, E. C.; \& Rubira, A. F. - E-polymer, 134, p.1 (2007).

14. Schneider, H. A. - J. Therm. Anal. Cal., 40, p.677 (1993).

15. Gupta, M. C. \& Viswanath, S. G. - J. Therm. Anal. Cal., 47, p.1081 (1996).

16. Pielichowski, J. \& Pielichowski, K. - J. Therm. Anal. Cal., 43, p.505 (1995).

17. Howell, B. A. \& Ray, J. A. - J. Therm. Anal. Cal., 83, p.63 (2006).

18. Núñez-Regueira, L.; Villanueva, M. \& Fraga-Rivas, I. J. Therm. Anal. Cal., 83, p.727 (2006).

19. Saha, B. \& Ghoshal, A. K. - Thermochim. Acta, 451, p.27 (2006).

20. Vyazovkin, S. \& Wight, C. A. - Thermochim. Acta, 340/341, p.53 (1999).

21. Vyazovkin, S. Thermochim. Acta, 355, p.155 (2000).

22. Friedman, H. J. Polym. Sci. C, 6, p.195 (1965).

23. Ozawa, T. - Bull. Chem. Soc. Jpn., 38, p.1881 (1965).

24. Flynn, J. H. \& Wall, L. A. - J. Res. Natl. Bur. Stand. Sect. A, 70, p.487 (1966).

25. Vyazovkin, S. \& Dollimore, D. J. Chem. Inf. Comput. Sci., 36, p.42 (1996).

26. Vyazovkin, S \& Sbirrazzuoli, N. - Macromol. Rapid Commun., 27, p.1515 (2006).

27. Vyazovkin, S. and Wight, C. A. - Ann. Rev. Phys. Chem., 48, p.125 (1997).

28. Aoyagi, Y.; Yamashita, K \& Doi, Y. - Polym. Degrad. Stab., 76, p.53 (2002).

29. Lecomte, H. A. and Liggat, J. J. - Polym. Degrad. Stab., 91, p.681 (2006).

30. Saha, B.; Maiti, A. K. \& Ghoshal, A. K. - Thermochim. Acta, 444, p.46 (2006).

31. Erceg, M.; Kovacic, T. \& Klaric, Y. - Polym. Degrad. Stab., 90, p.86 (2005).

Enviado: $17 / 10 / 09$

Reenviado: 04/02/10

Aceito: $11 / 02 / 10$

DOI: 10.1590/S0104-14282010005000023 